\title{
DOENÇA SEXUALMENTE TRANSMISSÍVEL PODE CAUSAR CÂNCER DE BOCA?
}

Catarina Luzia dos Santos José Monteiro

Graduanda em Odontologia pela Universidade do Grande Rio (UNIGRANRIO ),

Duque de Caxias, RJ, Brasil

catarinamonteiro@unigranrio.br

Ligia de Paula Barros Queiroz

Graduada em Enfermagem pela Faculdade União Araruama de Ensino (UNILAGOS), Araruama, RJ, Brasil queirozligia70@gmail.com

Jackeline Nogueira de Paula Barros

Mestre em Odontologia Clínica pela Universidade Federal do Rio de Janeiro (UFRJ),

Rio de Janeiro, Brasil jackelinenpb@gmail.com.br

\section{RESUMO}

O Papiloma Vírus Humano (HPV) é uma infecção sexualmente transmissível (IST), frequente em região ano-genital e rara em região bucal, porém devido a crescente prática do sexo oral essa prevalência vem aumentando, sendo atualmente considerada um grave problema de saúde pública, com elevada taxa de morbimortalidade e grande impacto psicossocial. Uma das manifestações clínicas orais do HPV é o Carcinoma e seu diagnóstico se faz através de exame clínico, citologia, biópsia, imuno-histoquímico e biologia molecular. Já o tratamento é variável e de acordo com o estágio em que a doença se encontra. Sendo assim, o presente estudo tem como objetivo avaliar a correlação entre o HPV e o Câncer de Boca através da revisão de literatura pesquisada nas principais bases de dados (Scielo, Lilacs e Pubmed) durante o período entre 2000 e 2018 tendo como palavras-chave: HPV, câncer de boca, carcinogênese e em inglês: HPV, oral cancer e carcinogenesis visando colaborar para a elaboração de políticas públicas de educação e prevenção mais eficazes, além do diagnóstico precoce e tratamento adequado para a melhora da qualidade de vida de todos os envolvidos.

Palavras-chave: HPH, Câncer de boca, Carcinogênese 


\title{
SEXUALLY TRANSMISSIBLE DISEASE CAN CAUSE MOUTH CANCER?
}

\begin{abstract}
The Human papillomavirus (HPV) is a sexually transmitted infection (STI), common in anogenital region and rare in the oral region. However, but due to the growing practice of oral sex, this prevalence is increasing and is currently considered a serious public health problem with great morbidity and mortality rate and great psychosocial impact. One of the oral clinical manifestations of HPV is Carcinoma and its diagnosis more serious than the clinical, cytology biopsy, immunohistochemistry and molecular biology. However, The treatment is variable and according to the situation in which the disease is. Thus, the present study aims to evaluate the correlation between HPV and Mouth Cancer through literature review performed in the main databases (Scielo, Lilacs and Pubmed) during the period between 2000 and 20018. The initial searc used the following keywords: HPV, câncer de boca, carcinogênese and in English: HPV, oral cancer and carcinogenesis aiming to collaborate for the elaboration of public education policies and prevention, in addition the early diagnosis and the appropriate treatment to improve the quality of life of the individuals involved.
\end{abstract}

Keywords: HPV. Oral cancer. Carcinogenesis.

\section{INTRODUÇÃO}

Dentre as lesões malignas da cavidade oral e orofaringe, o carcinoma epidermóide, também conhecido como espinocelular ou de células escamosas é o mais comum, com prevalência de aproximadamente $90 \%$ quando comparados aos outros tipos, cujos principais fatores de risco são o alcoolismo e tabagismo (MOTTA et al., 2009). Porém, estudos demonstram uma estreita relação entre o Vírus Papiloma Humano (HPV) e o Câncer de Boca e Orofaringe (SOBRAL et al., 2014).

O HPV possui em sua estrutura uma cadeia dupla de DNA que infecta os queratinócitos situados em epitélio de mucosa anogenital, boca e orofaringe de seres humanos, podendo causar doenças que variam desde uma verruga até o câncer (ZUR, 2009). A transmissão se dá através de relações sexuais sem proteção ou precocemente do trato genital da mãe para a cavidade bucal da criança, durante o nascimento (PYTYNIA et al., 2014).

Porém a maioria das infecções por HPV é autolimitada e com eliminação espontânea em aproximadamente dois anos, sem causar lesões e sintomas, pois sabe-se 
que somente a infecção pelo vírus não é suficiente para desenvolver o carcinoma (ZARDO GP et al., 2014), contudo o subtipo clínico HPV 16 é o mais prevalente e responsável por cerca de $50 \%$ de todos os cancros do colo do útero e $80-90 \%$ dos cancros da cabeça e pescoço positivos para o HPV (POLZ-GRUSZKA et al., 2015), atingindo proporções epidêmicas na última década, o que configura um problema de saúde pública mundial (MARUR et al., 2010).

Sendo assim, esse trabalho tem como objetivo avaliar a correlação entre o HPV e o Câncer de boca mediante uma atual revisão de literatura nas bases de dados Scielo, Pubmed e Lilacs, nos últimos vinte anos, usando como palavras-chave os termos em português: HPV, câncer de boca, Carcinogênese e em inglês: HPV, oral cancer e carcinogenesis, a fim de colaborar para a elaboração de políticas públicas de educação e prevenção mais eficazes, além do diagnóstico precoce e tratamento adequado para a melhora da qualidade de vida de todos os envolvidos.

\section{REVISÃO DE LITERATURA}

\subsection{HPV}

O HPV é um vírus DNA relativamente pequeno, não-envelopado, com $55 \mathrm{~nm}$ de diâmetro que apresenta tropismo por células epiteliais responsáveis pelo desenvolvimento de verrugas em diferentes grupos de mamíferos, inclusive no homem, e que podem causar infecções na pele e nas mucosas (LETO et al., 2011). Pode ser descrito em mais de 120 tipos em que os considerados carcinogênicos, através de evidências em estudos, são os HPV 16, 18, 31, 33, 35, 39, 45, 51, 52, 56, 58 e 59 (ZARDO GP et al., 2013). Em geral, a maioria das infecções por HPV é controlada pelo sistema imune e eliminada naturalmente pelo organismo, porém alguns persistem e podem causar lesões (MACHADO et al., 2004).

Segundo o Instituto Nacional de Ciência e Tecnologia das Doenças do Papilomavírus Humano (INCTDPH) em 2013, a transmissão pode ser realizada através do contato entre pele e mucosa infectada, e mesmo que raro e sem saber muitas das vezes que possui o vírus, o portador pode propagá-lo também por meio de contato com mão, pele, objetos, toalhas, roupas íntimas e até pelo vaso sanitário. Além disso, quando manifestada a doença, esta geralmente causa grande impacto psicossocial, normalmente acompanhado de problemas emocionais e psicológicos, relatados na maioria das vezes como sentimento de tristeza, vergonha e depressão podendo dessa forma retardar uma possível intervenção médica. 
Para o diagnóstico da doença, é preciso realização de um exame clínico detalhado para coleta da história do paciente e suas práticas, verificação de possíveis lesões e seu aspecto, assim como realização de biópsia, exame imuno-histoquímico para identificar características moleculares das doenças e teste de biologia molecular para presença do genoma dos HPV em células, tecidos e fluidos corporais (INCTDPH, 2013).

Estimativas da OMS indicam que 290 milhões de mulheres no mundo são portadoras do vírus, sendo $32 \%$ infectadas pelos tipos 16 e 18, os mais implicados com as neoplasias malignas, já para o INCTDPH cerca de mais de 10 milhões de pessoas possam ser portadoras do vírus HPV. Registra-se 700 mil novos casos a cada ano, sendo que $80 \%$ dentre a população sexualmente ativa vão contrair HPV durante a vida, provavelmente devido ao contato sexual relativamente cedo dos jovens, justificando assim estudos que mostram o Brasil entre países com maior prevalência da infecção por HPV no mundo. Por isso, em 2015 foi disponibilizado pelo SUS (Sistema Único de Saúde) para mulheres na faixa etária compreendida entre 9 e 26 anos que vivem com HIV/AIDS a vacina contra HPV, já que pessoas com o sistema imune comprometido são mais suscetíveis, e em 2017 para meninos de 12 e 13 anos, meninas de 14 anos e homens vivendo com HIV/AIDS na faixa etária entre 9 e 26 anos de idade, bem como para imunodeprimidos, transplantados e pacientes oncológicos, tendo como meta uma cobertura vacinal com a ampliação gradativa dessa faixa etária até o ano de 2020.

Vale ressaltar que, desde 2006 a Agência Nacional de Vigilância Sanitária do Brasil (ANVISA) regulamentou a comercialização da vacina quadrivalente e 2009 a bivalente, por ser um método eficaz e de relevante custo-benefício, para combater o HPV (ZARDO GP et al., 2013), podendo ser observada, desta forma, na tabela 1, características entre elas. 
Tabela 1 - Características das vacinas anti-HPV

\begin{tabular}{|l|c|c|}
\hline \multicolumn{1}{|c|}{ Característica } & Quadrivalente & Bivalente \\
\hline Composição/Tipo & VLP L1 HPV 6,11,16,18 & VLP L1 HPV 16,18 \\
\hline Nome comercial & $\begin{array}{c}\text { Vacina Quadrivalente Recombinante } \\
\text { contra Papilomavírus Humano }\end{array}$ & $\begin{array}{c}\text { Vacina contra HPV oncogê- } \\
\text { nico (16 e 18 Recombinante } \\
\text { com ASO4) }\end{array}$ \\
\hline Posologia & 0,5 mL IM 0, 2 e 6 meses & $0,5 \mathrm{~mL} \mathrm{IM} \mathrm{0,1} \mathrm{e} \mathrm{6} \mathrm{meses}$ \\
\hline Segurança Geral & Geralmente segura e bem tolerada & $\begin{array}{c}\text { Geralmente segura e bem } \\
\text { tolerada }\end{array}$ \\
\hline Gravidez & $\begin{array}{c}\text { Categoria B / não associada a mal- } \\
\text { formação, ou má evolução obsté- } \\
\text { trica }\end{array}$ & Segura e bem tolerada \\
\hline Lactação & Segura e bem tolerada & - \\
\hline Proteção cruzada & Eficácia (NIC 2/3 ou AIS) & Eficácia (infecção persistente) \\
\hline HPV 31/45 & $62 \%$ (95\% IC 10-85) & $60 \%$ (97,9\% IC 28-79) \\
\hline HPV 31/33/45/52/58 & 43\% (95\% IC 07-66) & $41 \%$ (97,9\% IC 20-56) \\
\hline $\begin{array}{l}\text { HPV31/33/35/39/45/ } \\
\text { 51/52/56/58/59 }\end{array}$ & $38 \%$ (95\% IC 06-60) & \\
\hline
\end{tabular}

Fonte: (Giraldo et al., 2008)

Em relação as consequências geradas pelo HPV, pode-se citar, segundo estudos epidemiológicos conduzidos por Tinhofer et al. (2015), que os carcinomas de células escamosas de cabeça e pescoço (CECP), pela exposição de fatores exógenos (consumo de tabaco e álcool) estão em declínio, enquanto o CECP dirigido por infecção pelo papilomavírus humano (HPV) está aumentando em indivíduos mais jovens nos últimos anos. D'Souza et al. (2009) concordam com tais achados na medida que afirmam ser um dos fatores que contribuiu para a elevação do grau de associação entre CECP e HPV foi o aumento do número de parceiros sexuais através de sexo ano-vaginal e oral, sendo as práticas sexuais orais mais difundidas entre adolescentes. Tais autores ainda chamam a atenção para o aumento do risco para outros tipos de cânceres, devido a comportamentos considerados de alto risco. Já Pottes em seus estudos em 2017, apontou uma grande vulnerabilidade entre idosos em relação as infecções sexualmente transmissíveis (IST's), devido principalmente as inovações tecnológicas farmacêuticas e da medicina associada à mudança comportamental que envolve a sexualidade na terceira idade.

Porém é importante salientar que, somente o tumor é comprovadamente diri- 
gido pelo HPV, se a expressão de oncogênese relacionados ao HPV for detectada (PRIGGE et al., 2017), pois estudos demonstram que os tumores HPV+ no nível molecular, provavelmente, possuem um prognóstico muito mais favorável, mesmo em estágios mais avançados (ANG et al., 2010). Além disso, as diferenças nas características biológicas e no prognóstico, leva a um melhor resultado na medida que possibilita a realização de uma terapia individualmente otimizada para esses pacientes minimizando a toxicidade relacionada ao tratamento (WANG et al., 2016).

Além das lesões malignas, como o câncer de boca, o HPV pode apresentar outras manifestações clínicas, de cunho benigno como: Hiperplasia Epitelial Focal, Condiloma Acuminado, Papiloma Escamoso, Verruga Vulgar, além da leucoplasia e líquen plano erosivo (NEVILE et al., 2009).

\subsubsection{MANIFESTAÇÃO CLÍNICA ORAL DO HPV}

\section{Câncer de Boca}

O câncer de boca é uma neoplasia maligna proveniente de um crescimento desordenado de células, cuja a transformação genética pode atingir outros sítios anatômicos, através da corrente sanguínea ou linfática formando novos tumores à distância (metástase), com grande impacto psicossocial, custos elevados e altas taxas de morbimortalidade, sendo considerada um problema de saúde pública mundial (PORCARO-SALLES, 2007; INCA, 2011).

A Organização Mundial de Saúde (OMS) considerou que o câncer foi uma das principais causas de morte em todo o mundo, responsável por 8,8 milhões de mortes em 2015, sendo $22 \%$ das mortes associadas a infecção pelo HPV e Hepatite em países de baixa e média renda. Esta mesma organização ainda estimou que, em 2018 serão 9,6 milhões de morte e em 2030 haverá aproximadamente 21,4 milhões de casos incidentes de câncer e 13,2 milhões de mortes, tornando-se esses números maiores em tais paises. (INCA 2014; PLUMMER et al., 2016; OMS, 2018). Já no Brasil ocorreram em 2015, cerca de 4.672 óbitos por câncer de cavidade oral em homens e 1.226 em mulheres (BRASIL, 2017).

Segundo o Instituto Nacional do Câncer (INCA) em 2018 e 2019 estima-se cerca de 11.200 novos casos de câncer de boca entre os homens, constituindo o quinto tipo mais frequente (principalmente na região Sudeste) e 3.500 novos casos em mulheres, representando o décimo segundo tipo mais frequente. Tais valores cor- 
respondem a um risco estimado de 10,86 casos novos a cada 100 mil homens e 3,28 a cada 100 mil mulheres (INCA, 2018). Todavia, para a American Cancer Society - ACS (2017) essa estimativa foi de 49.670 novos casos de câncer de boca e orofaringe, sendo duas vezes mais prevalente em homens do que em mulheres, com maior incidência entre pessoas de cor branca, quando comparados às negras, ocorrendo grande parte devido a associação com a infecção pelo HPV.

O carcinoma espinocelular (CEC), também chamado de células escamosas ou epidermóide (Figura 1), representa de 80 a $90 \%$ dos casos de neoplasias malignas diagnosticadas na boca, predominantemente no sexo masculino e na faixa etária da quinta e sexta décadas de vida (PIRES et al, 2013), sendo considerado o sexto câncer mais prevalente no mundo (SIEGAL et al., 2014). E para Neville e Damm (2016) é mais prevalente em região lingual e de assoalho de boca, porém pode ocorrer em qualquer sítio da mucosa bucal, como em mucosa jugal, retromolar, gengiva, palato mole e palato duro, sendo sua manifestação clínica variável, podendo apresentar-se como uma lesão ulcerada ou leucoplasica ou eritroplasica ou ainda uma combinação destas (Figura 1).

Figura 1 - Carcinoma de células escamosas

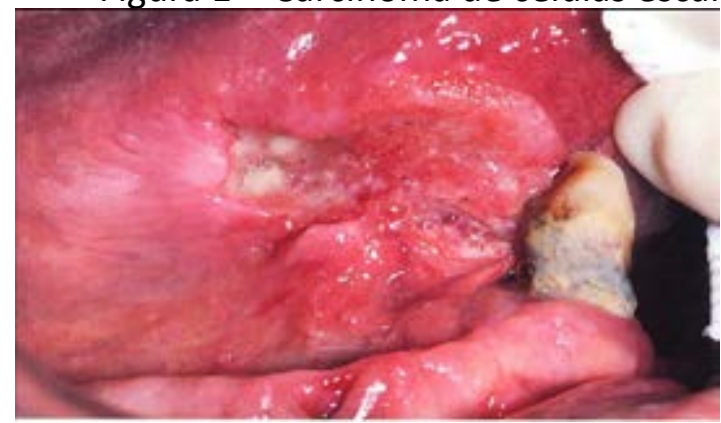

Fonte: (Neville B. W., Patologia Oral e Maxilofacial. 2009,

p.416)

A etiologia do câncer de boca é multifatorial, porém o principal fator de risco é o consumo do tabaco e em alguns países asiáticos o hábito de mascar betel (planta originária da Índia), todavia já está consolidado na literatura que a associação do uso do tabaco com o álcool pode elevar em mais de 35 vezes a chance do desenvolvimento do CEC, pois o álcool aumenta a permeabilidade da mucosa bucal aos agentes carcinogênicos presentes no tabaco (OLIVEIRA et al., 2013; PIRES et al., 2013; AMERICAN CANCER SOCIETY, 2017a).

Além disso, outros fatores considerados predisponentes são a exposição excessiva a radiação ultravioleta (RUV), com sua ação física deletéria agindo no 
DNA celular, comum em lábio inferior e dentre os agentes infecciosos, o Papilomavírus humano (HPV) que tem sido associado por estar relacionado a carcinomas de outras mucosas corporais. Ainda, pode-se citar dieta pobre em frutas e vegetais e má higiene bucal, que vêm sendo investigado quanto a sua implicação na carcinogênese, principalmente, do câncer de língua e orofaringe (OLIVEIRA et al., 2009; AMERICAN CANCER SOCIETY, 2017a).

Zardo et al. (2013), ainda citam como um cofator para a carcinogênese a predisposição genética, status imunológico, tabagismo, uso de contraceptivos orais, nível socioeconômico e coinfecções por Chlamydia trachomatis (bactéria causadora da clamidíase) e HIV. Já a American Cancer Society, 2017 inclui o excesso de peso corporal, além de fatores intrínsecos como fatores genéticos, condições hormonais e imunes. E para a OMS, 2018 o envelhecimento foi considerado outro fator fundamental para o desenvolvimento do câncer, provavelmente devido a acumulação do risco total combinada com os mecanismos de reparação celular menos eficazes típicos do enveIhecimento.

O diagnóstico baseia-se no exame clínico detalhado, anamnese minuciosa, bem como exames complementares, tendo como principais sinais e sintomas lesão em boca ou garganta com sangramento fácil e sem cura espontânea, mancha branca (leucoplásica) e/ou vermelha (eritroplásica), caroço ou espessamento na garganta, massa endurecida no pescoço, dor de ouvido, dificuldade de mastigar, engolir ou mexer a língua ou mandíbula, sendo tais sintomas frequentes num diagnóstico tardio. Entretanto a detecção precoce de anormalidades pré-malignas e cânceres se faz necessário para que o tratamento tenha um prognóstico mais favorável segundo a portaria № 1.008, DE 30 DE SETEMBRO DE 2015. (INCA, 2018).

Quanto ao tratamento, o objetivo principal é curar o câncer ou prolongar a vida do paciente e consiste em cirurgia, quimioterapia e radioterapia ou uma combinação destes

dependendo do tipo de protocolo individualizado adotado e do estadiamento (classificação) da doença, porém a imunoterapia vem sendo vista como uma nova opção de tratamento para canceres em fase avançada ou recorrente, compondo uma taxa de sobrevida aumentada em casos de indivíduos HPV positivo e na medida em que se faz o diagnóstico de forma mais precoce possível (AMERICAN CANCER SOCIETY, 2017a). Outro objetivo fundamental é a melhorara da qualidade de vida do paciente através de cuidados paliativos e apoio psicológico (OMS, 2018). 
Diante do exposto a prevenção é ainda o melhor caminho, através de uma vida saudável e livre de estresse, já que a vacinação contra o HPV para doenças genitais ainda deva ser melhor consolidada para doenças bucais e de orofaringe. Ja para a prevenção secundária, o autoexame de boca foi considerado uma ótima estratégia, desde que as pessoas sejam devidamente instruídas para isto (ALMEIRA et al., 2011), o que não é corroborado pelo INCA, pois a não recomendação do autoexame é devido ao risco que a população corre em negligenciar lesões potencialmente malignas, o que poderá levar ao diagnóstico tardio.

\section{HIPERPLASIA EPITELIAL FOCAL (HEF)}

A HEF ou doença de Heck é uma doença rara da mucosa bucal, benigna, que geralmente aparece na infância, claramente associada aos sorotipos HPV 13 e 32 (VERA-IGLESIAS, 2007), sem predileção de gênero e as partes interessadas locais incluem a mucosa labial, bucal e lingual. (ROSA, 2003). É clinicamente caracterizada por lesões bem definidas, que podem ser únicas ou múltiplas, como nódulos ou pápulas (figura 2), arredondadas ou planas, que freqüentemente coalescem (FERRARO, 2011).

Figura 2 - Hiperplasia epitelial focal

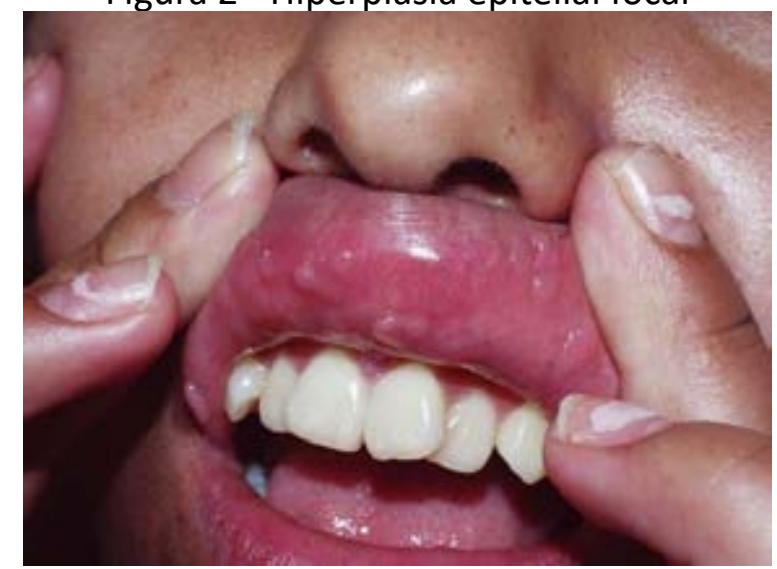

Fonte: (Neville B. W., Patologia Oral e Maxilofacial. 2009, p.368)

\section{CONDILOMA ACUMINADO}

Ao exame clínico o condiloma acuminado (figura 3) se manifesta como lesão superficial, única ou múltipla, de crescimento exofítico, de aspecto papilar, frondoso e róseo, distribuído de forma isolada ou coalescente, formando uma massa seme- 
Ihante à couve-flor. Geralmente as lesões são assintomáticas e alguma vezes regridem espontaneamente e podem ou não apresentar recidiva (CASTRO, 2004).

Figura 3 - Condiloma acuminado

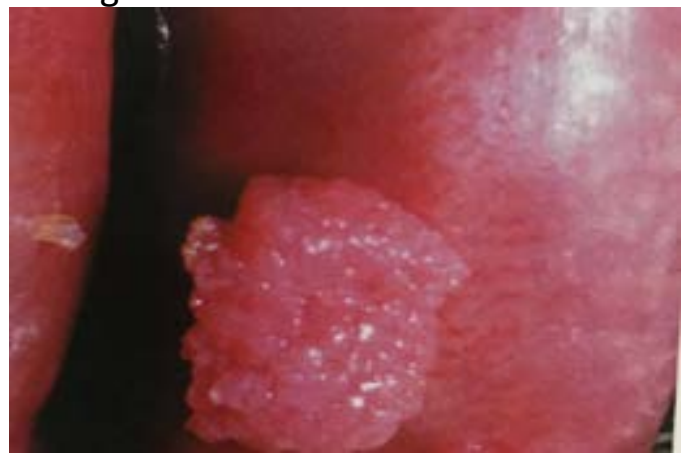

p.367)

Fonte: (Neville B. W., Patologia Oral e Maxilofacial. 2009,

\section{PAPILOMA ESCAMOSO}

O Papiloma Escamoso (figura 4) é um aumento benigna do epitélio escamoso estratificado (MENDES, 2015) que clinicamente se apresenta de forma exofítica de coloração e tamanho variado (OLIVEIRA, 2018).

Figura 4 - Papiloma escamoso

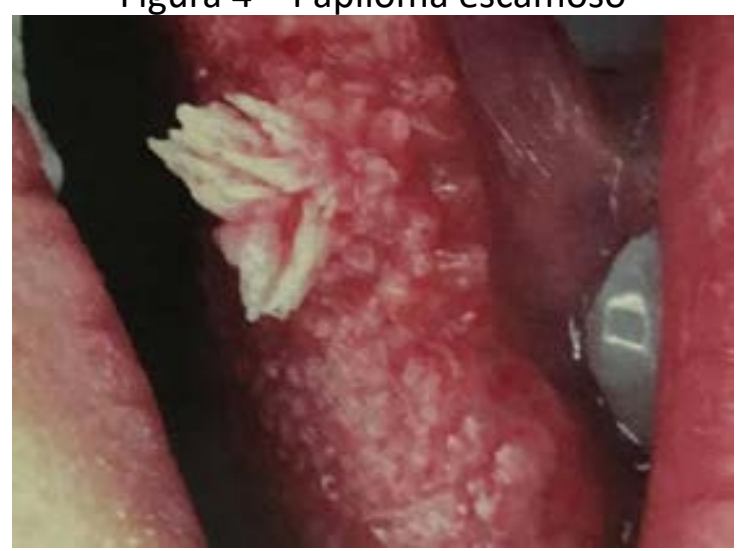

Fonte: (Neville B. W., Patologia Oral e Maxilofacial. 2009, p.367)

\section{LÍQUEN PLANO EROSIVO}

Como uma doença muco cutânea inflamatória crônica, o líquen plano erosivo (figura 5) é visto em sua forma clínica (NICO et al., 2011) como áreas atróficas e eritematosas geralmente cercadas por finas estrias radiantes (CERVANTES GARCIA DE SOUSA, 2008). 
Figura 5 - Líquen plano

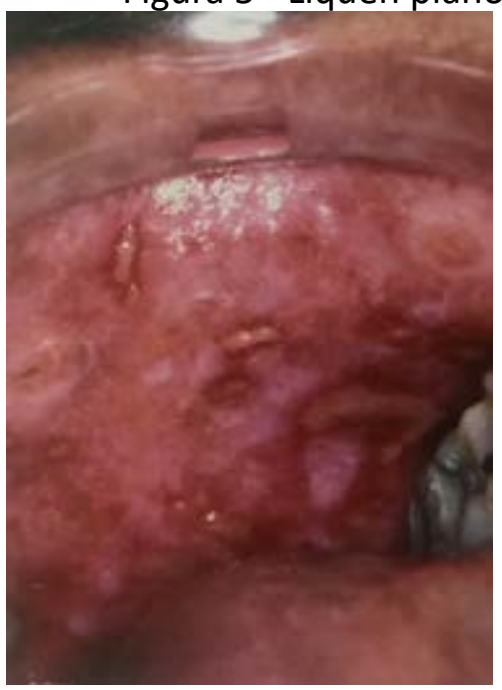

Fonte: Neville B. W., Patologia Oral e Maxilofacial. 2009,

p.790.

\section{VERRUGA VULGAR}

A verruga vulgar (figura 6) é uma hiperplasia focal, sem malignidade, sendo em seu aspecto, pápula ou nódulo indolor, apresentando projeções papilares ou uma superfície áspera semelhante a seixos (SIQUEIRA et al., 2016).

Figura 6 - Verruga vulgar

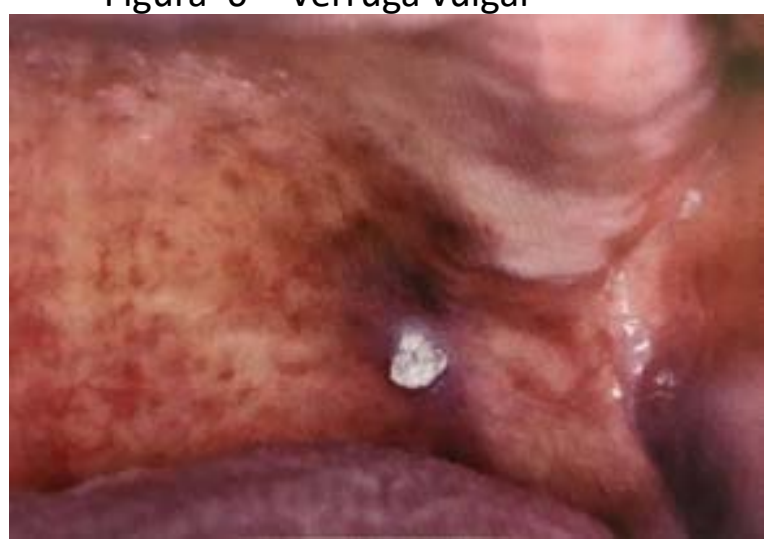

p.366).

Fonte: (Neville B. W., Patologia Oral e Maxilofacial. 2009,

\section{LEUCOPLASIA}

A leucoplasia (Figura 7) pode ser vista como uma placa predominantemente branca da mucosa bucal, que não se destaca à raspagem, cuja superfície pode apresentar-se lisa, rugosa ou verrucosa (RODRIGUES et al., 2000). 
Figura 7 - Lesão leucoplásica.

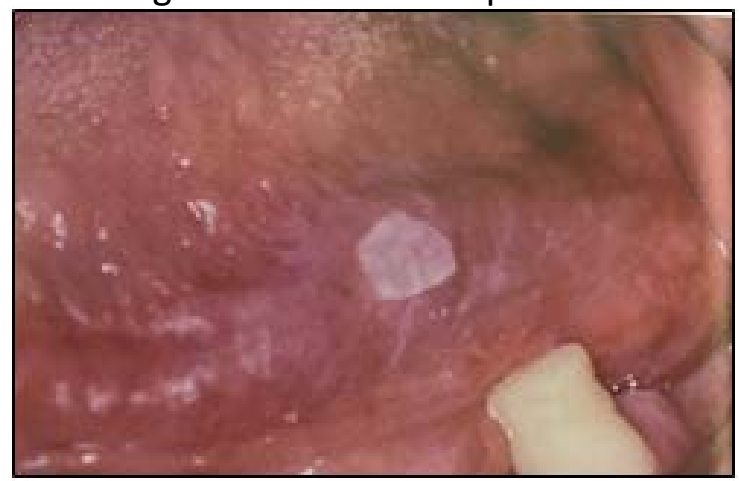

p.392.

Fonte: Neville B. W., Patologia Oral e Maxilofacial. 2009,

\section{CONSIDERAÇÕES FINAIS}

Sendo assim, esse estudo mostrou que, o câncer de boca é causado pela transformação de células normais em células tumorais e é o resultado da interação entre fatores genéticos do indivíduos e agentes externos cancerígenos como os físicos, químicos e biológicos, o qual se inclui o HPV, sendo a transmissão desse vírus realizada por meio do contato direto com a pele ou mucosa infectada através de práticas sexuais ano-genito-oral, sem prevenção ou precocemente de mãe para filho, durante o parto de mãe infectada. Por isso, o cuidado com a saúde geral, evitando os fatores de risco, uso de preservativo, vacinação, além de campanhas para conscientização da população, diagnóstico precoce e o acesso ao tratamento adequado em tempo hábil, são condições que podem diminuir significativamente esse problema de saúde pública no Brasil, meIhorando a qualidade de vida da população.

\section{REFERÊNCIAS}

ACS, American Cancer Society. Estadísticas importantes sobre los tipos de cáncer de orofaringe y de cavidad oral. 2018. Disponível em: <https://www.cancer.org/es/cancer/ cancer-de-orofaringe-y-de-cavidad-oral/acerca/estadisticas-clave.html>. Acesso em: 17 set. 2018.

ALMEIDA, Fernanda Campos Sousa de et al. Popularização do autoexame da boca: um exemplo de educação não formal-Parte II. Ciência \& Saúde Coletiva, v. 16, p. 1589- 
1598, 2011.

ANG, K. Kian et al. Human papillomavirus and survival of patients with oropharyngeal cancer. New England Journal of Medicine, v. 363, n. 1, p. 24-35, 2010.

CASTRO, T. M. P. G. et al. Manifestações orais associadas ao papilomavírus humano (HPV)

D'SOUZA, Gypsyamber et al. Oral sexual behaviors associated with prevalent oral human papillomavirus infection. The Journal of infectious diseases, v. 199, n. 9, p. 1263-1269, 2009.

FERRARO, Cíntia Tereza Lima et al. Infecção oral pelo HPV e lesões epiteliais proliferativas associadas. Jornal brasileiro de patologia e medicina laboratorial, v. 47, n. 4, p. 451-459, 2011.

GIRALDO, Paulo C. et al. PREVENÇÃO DA INFECÇÃO POR HPV E LESÕES ASSOCIADAS. DST-J bras Doenças Sex Transm, v. 20, n. 2, p. 132-140, 2008.

INCA, Instituto Nacional do Câncer. 27 de novembro - Dia Nacional de Combate ao Câncer. 2018. Disponível em: $\leq$ http://www.inca.gov.br/wcm/dncc/2015/dia-nacional-combate-cancer.asp >. Acesso em: 17 set. 2018.

INCA, Instituto Nacional do Câncer. ABC do câncer: Abordagens básicas para o controle do câncer. 2011. Disponível em: <http://bvsms.saude.gov.br/bvs/publicacoes/abc_do cancer.pdf $>$. Acesso em: 17 set. 2018.

INCA, Instituto Nacional do Câncer. Exames Complementares no Diagnóstico do Câncer. 2018. Disponível em: $\leq$ http://www.inca.gov.br/conteudo view.asp?ID=50 . Acesso em: 17 set. 2018.

Instituto Nacional de Câncer José Alencar Gomes da \& Silva. Estimativa 2018: Incidência de câncer no Brasil. Instituto Nacional de Câncer José Alencar Gomes da Silva (INCA, 2017). 
LETO, Maria das Graças Pereira et al. Infecção pelo papilomavírus humano: etiopatogenia, biologia molecular e manifestações clínicas. Anais Brasileiros de Dermatologia, 2011.

MACHADO, Paulo RL et al. Mecanismos de resposta imune às infecções Immune response mechanisms to infections. An Bras Dermatol, v. 79, n. 6, p. 647-664, 2004.

MARUR, Shanthi et al. HPV-associated head and neck cancer: a virus-related cancer epidemic. The lancet oncology, v. 11, n. 8, p. 781-789, 2010.

MARUR, Shanthi et al. HPV-associated head and neck cancer: a virus-related cancer epidemic. The lancet oncology, v. 11, n. 8, p. 781-789, 2010.

MELO FILHO, Mário Rodrigues de et al. Quality of life of patients with head and neck cancer. Brazilian journal of otorhinolaryngology, v. 79, n. 1, p. 82-88, 2013.

MENDES, Patricia Albuquerque. PAPILOMA ESCAMOSO: Relato de caso. REVISTA INCELÊNCIAS, v. 5, n. 1, 2015.

MICHELS, Karin B.; ZUR HAUSEN, Harald. HPV vaccine for all. The Lancet, v. 374, n. 9686, p. 268-270, 2009.

MOTTA, Rafael Da Ros et al. Ki-67 and p53 correlation prognostic value in squamous cell carcinomas of the oral cavity and tongue. Brazilian journal of otorhinolaryngology, $v$. 75, n. 4, p. 544-549, 2009.

NAKAGAWA, Janete Tamami Tomiyoshi; SCHIRMER, Janine; BARBIERI, Márcia. Vírus HPV e câncer de colo de útero. Revista Brasileira de Enfermagem, 2010.

NEVILLE, Brad W.; DAY, Terry A. Oral cancer and precancerous lesions. CA: a cancer journal for clinicians, v. 52, n. 4, p. 195-215, 2002. 
NEVILLE, Brad. Patologia oral e maxilofacial. Elsevier Brasil, 2016.

NICO, Marcello Menta Simonsen et al. Líquen plano oral. Anais Brasileiros de Dermatologia, v. 86, n. 4, p. 633-643, 2011.

Odontoestomatología, v. 19, n. 5, p. 239-247, 2003.

OLIVEIRA, Angélica Karolyne Mendonça et al. P o16-Papiloma escamoso em língua-diagnóstico e tratamento. ARCHIVES OF HEALTH INVESTIGATION, v. 6, 2018.

PIRES, F.R. et al. Oral squamous cell carcinoma: clinicopathological features from 346 cases from a single oral pathology service during an 8-year period. J Appl Oral Sci, v.21,n.5, p.460- 467, 2013.

PLUMMER, Martyn et al. Global burden of cancers attributable to infections in 2012: a synthetic analysis. The Lancet Global Health, v. 4, n. 9, p. e609-e616, 2016.

POLZ-GRUSZKA, Dorota et al. EBV, HSV, CMV and HPV in laryngeal and oropharyngeal carcinoma in Polish patients. Anticancer research, v. 35, n. 3, p. 1657-1661, 2015.

PORCARO-SALLES, J.M. Câncer de boca: uma visão multidisciplinar. 1ed. Belo Horizonte: Coopmed; 2007. p. 1-322.

POTTES, Fábia Alexandra et al. Aids e envelhecimento: características dos casos com idade igual ou maior que 50 anos em Pernambuco, de 1990 a 2000. Revista brasileira de epidemiologia, v. 10, p. 338-351, 2007.

PRIGGE, Elena-Sophie et al. Diagnostic accuracy of p16INK4a immunohistochemistry in oropharyngeal squamous cell carcinomas: A systematic review and meta-analysis. International journal of cancer, v. 140, n. 5, p. 1186-1198, 2017.

PYTYNIA, Kristen B.; DAHLSTROM, Kristina R.; STURGIS, Erich M. Epidemiology of HPV- 
associated oropharyngeal cancer. Oral oncology, v. 50, n. 5, p. 380-386, 2014.

RODRIGUES, Tânia Lemos Coelho et al. Oral leukoplakias: clinical-histopathologic relation. Pesquisa Odontológica Brasileira, v. 14, n. 4, p. 357-361, 2000.

RODRIGUES, Tânia Lemos Coelho et al. Oral leukoplakias: clinical-histopathologic relation. Pesquisa Odontológica Brasileira, v. 14, n. 4, p. 357-361, 2000.

ROSA, L. N. et al. Hiperplasia epitelial focal:¿ por qué enfermedad de Heck?. Avances en

SIEGEL, Rebecca; DESANTIS, Carol; JEMAL, Ahmedin. Colorectal cancer statistics, 2014. CA: a cancer journal for clinicians, v. 64, n. 2, p. 104-117, 2014.

SILVA, Maxwell Arouca et al. Câncer de boca: ação educativa centrada na capacitação para o autoexame. Revista Ciência em Extensão, v. 14, n. 1, p. 116-124, 2018.

SIQUEIRA, Taiane Siqueira et al. Estudo Retrospectivo das Infecções Virais em Cavidade Oral Diagnosticadas pelo Laboratório de Patologia Bucal da Universidade Federal de Santa Catarina (LPB-UFSC) em 10 Anos. 2016.

SOBRAL, Ana Paula Veras; ALMEIDA, Híttalo Carlos Rodrigues de; FONTES, Jéssica Pereira de Sá. Correlação do Papilomavírus Humano com o Carcinoma epidermoide Bucal: revisão sistemática. Revista de Cirurgia e Traumatologia Buco-maxilo-facial, v. 14, n. 2, p. 95-102, 2014.

TINHOFER, I. et al. Contribution of human papilloma virus to the incidence of squamous cell carcinoma of the head and neck in a European population with high smoking prevalence. European Journal of Cancer, v. 51, n. 4, p. 514-521, 2015.

VERA-IGLESIAS, E. et al. Hiperplasia epitelial focal. Actas dermo-sifiliograficas, v. 98, n. 9, p. 621-623, 2007. 
WANG, Xiaohong et al. Oncogenic HPV infection interrupts the expression of tumor-suppressive miR-34a through viral oncoprotein E6. Rna, 2009.

World Health Organization [Internet]. Human papillomavirus (HPV) and cervical cancer. 2016 [cited 2018 Set 16]. Available from: http://www.who.int/mediacentre/factsheets/ fs380/en/

ZARDO, Geisa Picksius et al. Vacina como agente de imunização contra o HPV. Ciência \& Saúde Coletiva, v. 19, p. 3799-3808, 2014. 